\title{
Application of SPWM Technology in Ground Resistance Tester
}

\section{LIU Hongzheng ${ }^{1, ~ a, ~ Y U A N ~ H a i y a n ~ 1, ~ a ~, ~ S H I ~ Z h e n ~}{ }^{2, ~ b}$, SUI Shougang ${ }^{2, ~ b}$, HU Xianzhe $e^{2, b}$,}

${ }^{1}$ State Grid Shandong Electric Power Research Institute, China

${ }^{2}$ Robotics and Intelligent Systems Research Institute of Shandong Jianzhu University, China a260347573@qq.com, bszxx307@126.com

\begin{abstract}
Keywords: SPWM; signal transmission; non-contact detection; ground resistance
Abstract. In order to meet the requirement of sine excitation signal for the test system of the free-wire ground resistance, based on the discussion of SPWM(Sinusoidal Pulse Width Modulation) technology, this paper summarizes the advantages of using SPWM to realize the transmission of energy signals, and the feasibility is verified by application test. This method is simple and has good driving force and maneuverability. This study provides a tool for non-contact detection method.
\end{abstract}

\section{Introduction}

In the test system of the free-wire ground resistance, it is necessary to transmit a certain sine excitation signal through the voltage transformer to the lead wire. In order to avoid the interference for the measurement result caused by $50 \mathrm{~Hz}$ power frequency and its higher harmonic resistance, we usually increase the current to improve the measuring the signal-to-noise ratio of the system, such as measure by a frequency different from power frequency. This method can further improve the measurement conditions and improve the measurement accuracy. The traditional method to measure the grounding resistance is to use the DDS circuit as the sine signal source, and use the power amplification circuit of driving voltage transformer. This method needs complicated hardware circuit, and it cannot provide larger excitation signal because of the low power utilization, high heating value 、 limited driving ability.

With the rapid development of power electronic technology and embedded technology, inverter control circuit develops from analog circuit to MCU control circuit and even digital signal processor $(\mathrm{DSP})^{[1]}$. Power output is generally used by IGBT, MOSFET and other easy-controlling switch device, and inverter device is toward the direction of smaller volume, higher efficiency , superior performance. It is a feasible plan to directly output sinusoidal excitation signal through DC inversion in the test system of the free-wire ground resistance. SPWM (Sinusoidal PWM) is a mature and widely used method of DC inverter. An important conclusion in the theory of sampling control is that when the pulse with equal pulse and different shape adds to the inertial link, the output waveform is basically the same. SPWM is based on this conclusion as the theoretical basis, and it uses the PWM wave which pulse width changes as sine wave to control the switch output circuit. PWM method's pulse voltage of the output area is equal with the output area of the sine wave by changing the frequency and amplitude the modulation wave to adjust the output voltage of the frequency and amplitude ${ }^{[2.8]}$. The portable grounding resistance measuring instrument is battery powered, its signal excitation unit is essentially an inductance coil with a certain inertia. Therefore, adopting SPWM technology is one of the effective means to realize the sine excitation signal emission.

Compared with the hardware power amplifier, SPWM has many incomparable advantages:

(1) Hardware function: The voltage amplification of the hardware power amplifier is not easy to adjust, and the signal parameters produced by the SPWM technology are easy to adjust and have better operability. 
(2) Hardware cost: Hardware controller and switch power amplifier circuit is complex while the SPWM method can be realized only by a PWM unit drive circuit. Therefore SPWM speeds up the speed of the peripheral circuit design and greatly reduces the design cost.

(3) Technical content: SPWM technology has a high theoretical level, thus improving the level of intelligent instrument.

\section{Working principle of SPWM power signal drive circuit}

The schematic diagram of the design of SPWM power signal drive circuit is shown in figure 1.

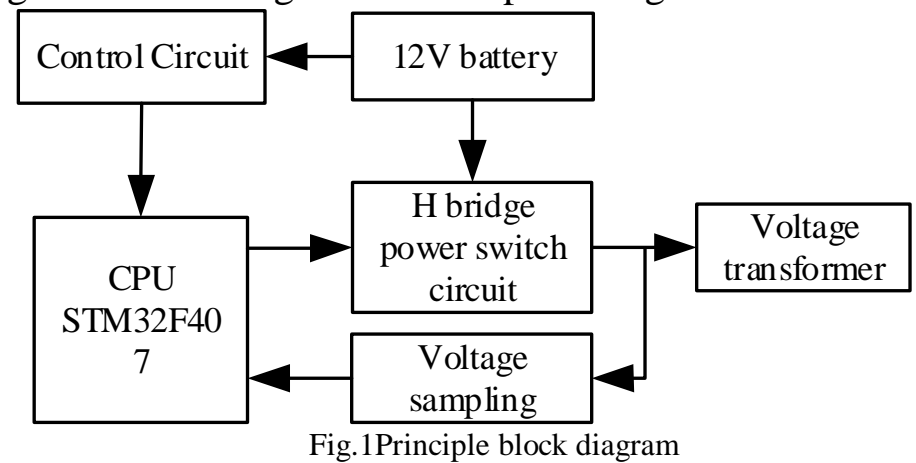

This design uses high-performance 32 bit microcontroller STM32F407 as the control unit, uses the $12 \mathrm{~V}$ lithium battery power supply to the whole system. The $\mathrm{H}$ bridge power switch circuit uses MOSFET as the power device, and works in the switching mode. In order to effectively avoid the same side of the bridge arm double tube conduction at the same time to burn out power and power devices, the circuit increases the half bridge driver chip IR2104S. The power switch circuit diagram of the $\mathrm{H}$ bridge is shown in figure 2 .

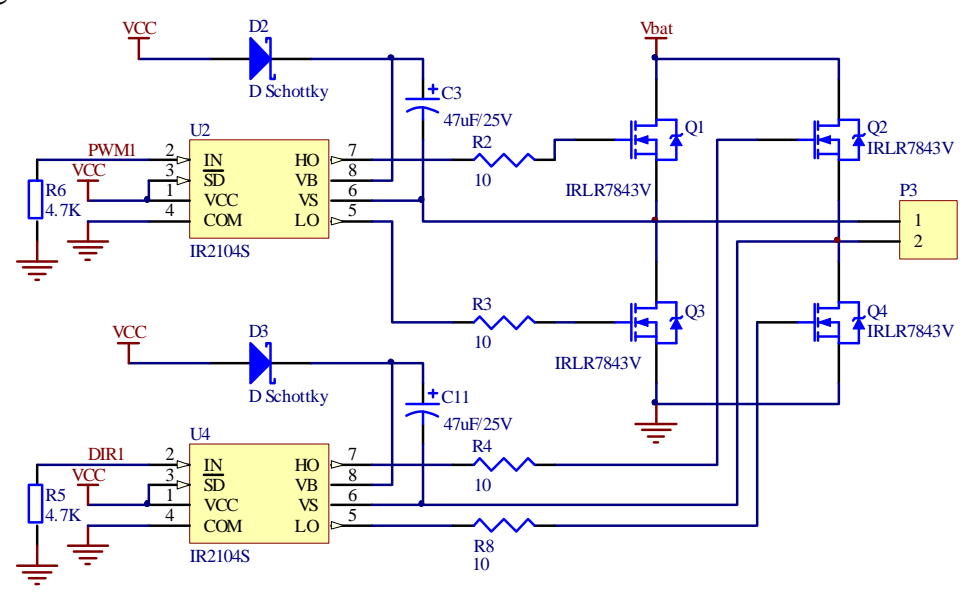

Fig.2 Power switch circuit diagram

In order to make the transformer get AC signal, the P3 terminal connected to the voltage transformer must make a pair of MOS tubes on the two diagonal lines alternating. When the Q1 tube and the Q4 tube is conducted, the current from the positive power supply by Q1 through the transformer, then it runs through Q4 back to the cathode of the power supply. When Q2 and Q3 are turned on, the current from the positive power supply by Q2 through the transformer, then runs through the Q3 back to the cathode of the power supply. So the voltage transformer can receive AC signal.

According to the introduction, the main problem of generating SPWM signals lies in the SPWM pulse width control method. When the sine wave is modulated, the triangular wave is used as carrier as a commonly used modulation method in the inverter field with less signal harmonic component. But the modulation method needs to be realized by analog circuits, while its design is relatively complex. Though the computer can achieve this design, the calculation formula for the transcendental equation is difficult to solve. So it is not suitable for real-time control of ordinary SCM[1.4]. 
In the single chip microcomputer control mode, the common method is to calculate sine wave data table by equal area method. A half sine wave is divided into $\mathrm{N}$ parts, and each part of the sine curve in the horizontal area is replaced with a equal height rectangular pulse. The midpoint of the rectangular pulse coincides with the midpoint of each part of the sine wave, so the rectangular pulse which consists with $\mathrm{N}$ equal amplitude and unequal width waveform is equal with half sine wave[3]. When the output signal parameters is relatively fixed, we can also receive data by computer and send data table into MCU ROM. MCU control PWM by looking up data table, and this method can greatly reduce the computational burden of MCU[8]. The test signal frequency of the grounding resistance test system is relatively fixed, so the look-up table method is used to obtain the SPWM pulse width data.

This method divides sine signal into $N$ equal parts (FM ratio) for $\frac{2 \pi}{N}$ radian. When $N$ is large enough, in order to reduce the difficulty of calculation, the approximate method for calculating the $\mathrm{K}$ parts of the pulse duty factor $n(k)$ are as follow :

$$
\begin{aligned}
n(k) & =M \times\left[A_{k}+\sin \left(\frac{2 \pi k}{N}\right)\right] \\
A_{k} & =0 \ldots \ldots \ldots . .\left(k<\frac{N}{2}\right) \\
A_{k} & =1 \ldots \ldots \ldots . .\left(k \geq \frac{N}{2}\right)
\end{aligned}
$$

In this design, we make use of modulation ratio $\mathrm{M}$ to adjust the signal amplitude to meet the system requirements for the amplitude of the signal.

And the pulse duty factor formula is:

$$
n(k)=\frac{t_{o n}(k)}{T_{c}}
$$

Where $T_{c}$ is the PWM duty factor when the pulse width data is $100 \% . t_{o n}(k)$ is the parameter controlling PWM duty factor as the k part which can be calculated by EXCEL to obtain the C data table. And this is the sine wave data table we need.

\section{Application experiment of SPWM technology in tower grounding resistance test system}

The experimental platform is based on the tower grounding resistance test system in the Institute of robotics and intelligent systems in Shandong Jianzhu University. Mutual inductance principle is used in the test system, and the voltage transformer and current transformer in this system are respectively clamped in the tower under the guidance of the line. The current transformer inducts current in lead line, meanwhile the voltage transformer in rod tower grounding inducts a specific voltage signal. The two signals are converted into digital $\mathrm{AD}$ into the MCU unit, and finally the grounding resistance value of the measured tower is obtained by a specific calculation method.

According to the above theoretical analysis, taking into account the processing speed of STM32F407, we take the FM ratio $\mathrm{N}$ as 256 , modulation ratio $\mathrm{M}$ as 1 . Data table obtained from EXCEL, and part of the data as shown in table 1. 
Tab.1 Sine data table

\begin{tabular}{lll}
\hline $\begin{array}{l}\text { Serial } \\
\text { number } \\
k\end{array}$ & $\begin{array}{l}t_{\text {on }}(k) \\
\text { Decimal }\end{array}$ & $\begin{array}{c}t_{\text {on }}(k) \\
\mathrm{HEX}\end{array}$ \\
\hline 0 & 0 & 0 \\
\hline 1 & 6.258013167 & 6 \\
\hline 2 & 12.51225674 & $\mathrm{C}$ \\
\hline 3 & 18.7589634 & 12 \\
\hline 4 & 24.99437036 & 18 \\
\hline 5 & 31.21472165 & $1 \mathrm{~F}$ \\
\hline 6 & 37.41627035 & 25 \\
\hline 7 & 43.5952809 & $2 \mathrm{~B}$ \\
\hline 8 & 49.74803128 & 31 \\
\hline 9 & 55.8708153 & 37 \\
\hline 10 & 61.95994484 & $3 \mathrm{D}$ \\
\hline 11 & 68.01175202 & 44 \\
\hline 12 & 74.02259147 & $4 \mathrm{~A}$ \\
\hline 13 & 79.98884248 & $4 \mathrm{~F}$ \\
\hline 14 & 85.90691121 & 55 \\
\hline 15 & 91.77323282 & $5 \mathrm{~B}$ \\
\hline$f$ & $7 \times$ &
\end{tabular}

We can restore the waveform by EXCEL drawing, and the effect diagram is shown in figure 3.

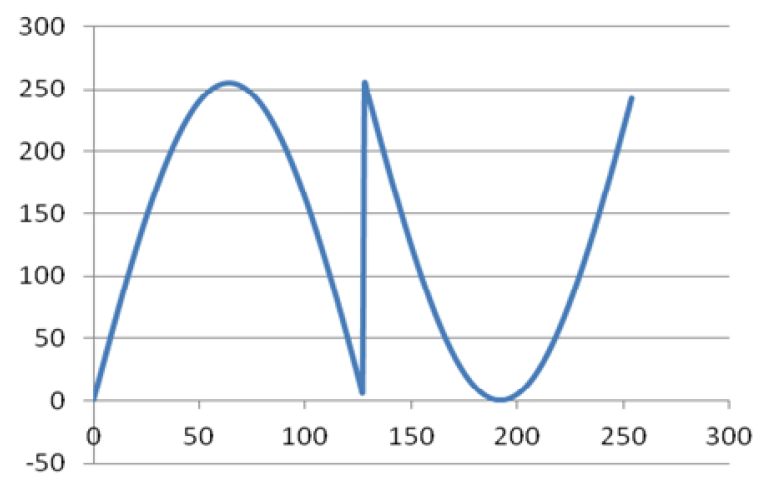

Fig. 3 Sine data table reduction waveform

As shown in Figure 3, the sine data table basically meets the requirements. In order to reduce the switching loss as much as possible and prolong the service life of the MOS tube, the unipolar modulation method is used to drive the MOS tube[6]. The Q1 and Q3 tube are set as the high frequency arm, and the Q2 and Q4 are the low frequency arm. The duty factor of the PWM signal is controlled by programming, and the Q1 tube and the Q3 tube of the $\mathrm{H}$ bridge are driven by the PWM signal. DIR1 signal controls the direction by another bridge arm of the Q2 tube and the Q4 tube. When the output is half wave, DIR1 control Q4 conduction; when the output negative half wave, DIR1 control Q2 conduction.

The PWM1 signal and the DIR1 signal are shown in figure 4. The red signal is PWM1 signal, and the green signal is DIR1 signal. 


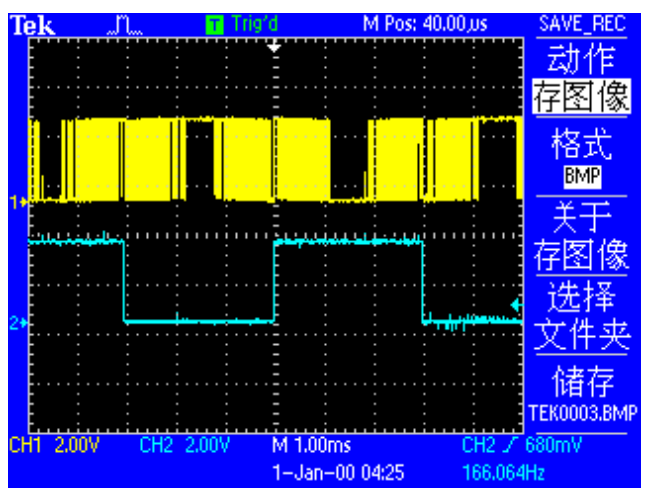

Fig.4 PWM1 and DIR1 signal

The two signals are loaded in the $\mathrm{H}$ bridge power module, whereby the voltage signal output at the $\mathrm{P} 3$ terminal is shown in figure 5.

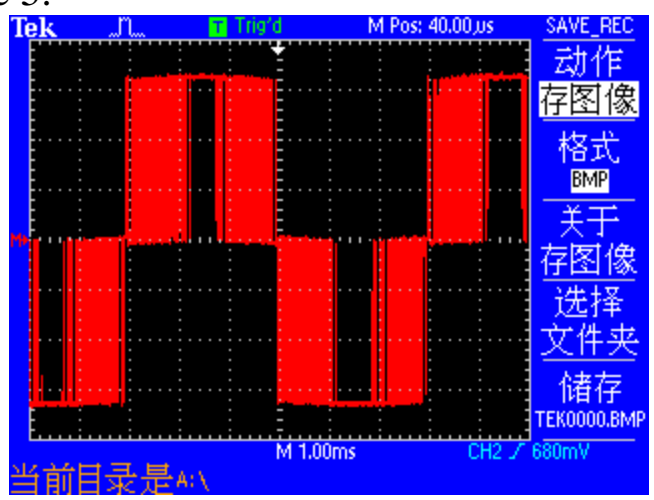

Fig. $5 \mathrm{H}$ bridge output signal

After the SPWM power signal passes through the inertial unit voltage transformer, the sinusoidal excitation signal with controllable parameters can be obtained on the two side of the transformer.

\section{Conclusion}

In this paper, a SPWM sine excitation signal control method based on look-up table is realized by using STM32F407 as the control core. And its feasibility is proved by the application in the tower grounding resistance test system.

Through the test, it can be found that the best output frequency waveform can be obtained as long as the best frequency modulation ratio is obtained by observing the test results. So the SPWM technology can be applied to the tower grounding resistance testing system. In order to take into account the output effect and the MCU resources, the method of frequency modulation ratio needs to be further studied.

\section{References}

[1] Chen Mingxing , Shi Bin , Chen Yiguo , et al.. A Novel Sampling Method of SPWM Based on DSP[J]. Electrical Automation, 2011,33(5):45-46.

[2] Chen Guocheng,Cai Liqing. Mathematical analysis on saddle waveform PWM based on isosceles triangle carrier wave[J]. Advanced Technology of Electrical Engineering and Energy | Adv Techno Electoral Energ,2015,34(9) :2-16.

[3] Wan Qiuyi, Li Shilin. Realization Based on Sequential Control the SPWM) [J]. Nuclear Electronics \& Detection Technology, 2011,31(5): 588-590.

[4] Chen Guocheng, Cai LIqing ). Mathematical analysis on SPWM and SAPWM based on sawtooth carrier wave ) [J]. Advanced Technology of Electrical Engineering and Energy |Adv Techno Electoral Energ,2015,34(10) :1-9. 
[5] Chen Xiaoou, Xu Chunming. The Key Problems of Three Level Inverter SVPWM in the 60 Degrees Corrdinates[J]. Advanced Technology of Electrical Engineering and Energy | Adv Techno Electoral Energ,2017,36(2):43-49.

[6] Chen Guocheng, Cai Liqing. Mathematical analysis on least switching PWM based on sawtooth carrier wave[J]. Advanced Technology of Electrical Engineering and Energy | Adv Techno Electoral Energ,2015,34(11):1-10.

[7] Huang Jun, Wang Zhaoan. Power electronic converter technology[M]. Beijing: China Machine Press, 1998.

[8] Zhao Qing ) . Study of a sine wave inverter based on unipolar SPWM control[D]. Hangzhou: Zhejiang University,2004. 